\title{
In vitro measurement of ambient pressure changes using a realistic clinical setup
}

\author{
Andersen, Klaus Scheldrup; Jensen, Jørgen Arendt
}

Published in:

Proceedings - IEEE Ultrasonics Symposium

Link to article, DOI:

10.1109/ULTSYM.2008.0264

Publication date:

2008

Document Version

Publisher's PDF, also known as Version of record

Link back to DTU Orbit

Citation (APA):

Andersen, K. S., \& Jensen, J. A. (2008). In vitro measurement of ambient pressure changes using a realistic clinical setup. In Proceedings - IEEE Ultrasonics Symposium (pp. 1096-1099). IEEE.

https://doi.org/10.1109/ULTSYM.2008.0264

\section{General rights}

Copyright and moral rights for the publications made accessible in the public portal are retained by the authors and/or other copyright owners and it is a condition of accessing publications that users recognise and abide by the legal requirements associated with these rights.

- Users may download and print one copy of any publication from the public portal for the purpose of private study or research.

- You may not further distribute the material or use it for any profit-making activity or commercial gain

- You may freely distribute the URL identifying the publication in the public portal

If you believe that this document breaches copyright please contact us providing details, and we will remove access to the work immediately and investigate your claim 


\title{
In vitro measurement of ambient pressure changes using a realistic clinical setup
}

\author{
Klaus Scheldrup Andersen and Jørgen Arendt Jensen \\ Center for Fast Ultrasound Imaging, Department of Electrical Engineering, \\ Bldg. 349, Technical University of Denmark, 2800 Kgs. Lyngby, Denmark
}

\begin{abstract}
Many attempts to find a non-invasive procedure to measure the local blood pressure have been made. In the last decade independent experiments have indicated that the amplitude of the subharmonic response from contrast agents is sensitive to the ambient pressure. This paper presents a new experimental setup for measuring the acoustic response of a contrast agent when subjected to ambient over pressure. The setup is very flexible offering completely arbitrary excitation and data acquisition, fast and accurate ambient pressure control, and precise timing. More importantly, it resembles a realistic clinical setup using a single array transducer for transmit and receive. In this experiment, the acoustic response of SonoVue (Bracco, Milano, Italy) was measured twice at six different ambient hydrostatic pressures in the interval 0 to $25 \mathrm{kPa}$ with an accuracy within $0.5 \mathrm{kPa}$. The acquired RF data was filtered and beamformed before further processing. To compensate for variations in bubble response and to make the estimates more robust, the relation between the energy of the subharmonic and the fundamental component was chosen as a measure over the subharmonic peak amplitude. The results of the first measurement sequence show an ambient pressure sensitivity of $0.42 \mathrm{~dB} / \mathrm{kPa}$ having a linear correlation coefficient of 0.94 . In the second sequence, a sensitivity of $0.41 \mathrm{~dB} / \mathrm{kPa}$ with a correlation coefficient of 0.89 was found.
\end{abstract}

\section{INTRODUCTION}

Finding a noninvasive and reliable approach to measure the human blood pressure locally in the body would provide doctors with a new tool to diagnose diseases related to the blood pressure. A noninvasive approach, which is based on flow estimation and a modification of the Bernoulli equation, already exists [1]. This gives an estimate of the pressure gradient, but was concluded not to provide reliable or reproducible results by Strauss et al. [2] and Reddy et al. [3]. Another existing procedure is to insert a pressure sensor directly into the vessel by means of a catheter. However, the presence of a pressure sensor inside the vessel will change the flow and, thereby, the blood pressure. Moreover, as this is an invasive approach, it is inconvenient to the patient and accompanied with a risk of infection.

Because of the high compressibility of gas, microbubbles containing air or gas can be used as local pressure sensors [4], [5], [6]. The idea of using an ultrasound contrast agent (UCA) to measure the cardiac pressure noninvasively was first proposed by Fairbank and Scully [4] in 1977. They claimed that the acoustic properties of the microbubbles change when the size of the bubbles change. To measure these size dependent oscillations, they suggested to measure the shift in resonance frequency but the results were, however, inconclusive. Other suggestions to measure the resonance shift at that time were made by Tickner [7] in 1982, Ishihara [8] in 1988, and Schlief and Poland [9] in 1993. Another approach was presented by Newhouse and Shankar [10], [11] in 1986. They showed theoretically and experimentally that accurate bubble size measurements are possible using a double frequency technique for determination of the sum and difference frequencies. The rapid dissolution time of free air bubbles prevented, however, any practical implementation at that time.

Since the introduction of the more stable second generation UCAs, new attempts to take advantage of the ambient pressure dependent acoustic properties have been initiated. In 1999, Bouakaz et al. [12], [13] presented an approach for measuring the disappearance time of free bubbles, which were generated at the region of interest by rupturing the contrast agent microbubbles using a low-frequency high acoustic amplitude pulse. Despite successful in vitro experiments and suggestions for further sensitivity improvements, no in vivo results or further investigations using this approach have been presented yet. Around the same time, Shi et al. [6] observed from experiments, using two single element transducers, that the subharmonic component of Levovist is highly sensitive to ambient pressure changes compared to the fundamental and the second harmonic component. They reported a $9.9 \mathrm{~dB}$ linear decrease of the peak amplitude of the subharmonic component when increasing the ambient pressure from 0 to $24.8 \mathrm{kPa}(1 \mathrm{kPa}=7.5 \mathrm{mmHg})$. Recently, the same group have presented similar results for Sonazoid, which was found to have an average decrease of $13.3 \mathrm{~dB}$ [14]. Furthermore, in 2005 they presented in vivo results for proof of concept of the capabilities of the subharmonic response [15]. However, as the measurements were performed directly on the abdominal cavity and the aorta by incision of two dogs, this can hardly be characterized as noninvasive. Also in 2005, Adam et al. [16] did a thorough and interesting study to understand the mechanisms of acoustic scattering and attenuation of Optison when subjected to ambient over pressure. One of the conclusions confirmed that the subharmonic of the transmitted frequency can be used to detect ambient pressure variations. Andersen and Jensen [17] has recently performed a parameter study to optimize the subharmonic sensitivity to ambient over pressure and found two very clear tendencies. First, the linear reduction of the subharmonic component, or the pressure sensitivity, is dependent on the acoustic driving pressure and peaks when in the upper end of the growth stage, which occurs when the 


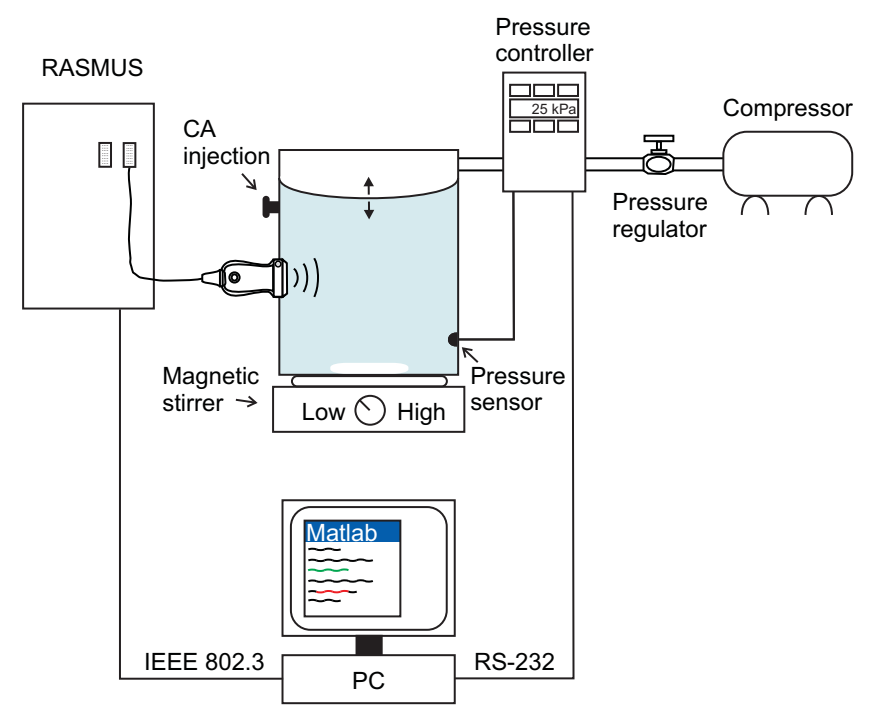

Fig. 1. Block diagram of the measurement setup. The left part shows the ultrasound acquisition part. The right part illustrates the pressure management system.

acoustic driving pressure causes the subharmonic to increase rapidly from background noise level to clearly visible in the spectrum. Second, the investigation also showed a clear relation between ambient pressure sensitivity and the length of the driving pulse.

As a setup using two transducers is not optimal in a clinical setting [16], this paper presents an experimental setup for measuring the fundamental and subharmonic response of a contrast agent when subjected to ambient over pressure, which more realistic resembles a clinical setting. The setup has been used to measure the pressure sensitivity of SonoVue (Bracco, Milano, Italy) using a standard ultrasound acquisition procedure and signal processing steps, which can easily be implemented in any commercial ultrasound scanner.

\section{Method}

\section{A. Experimental setup}

A block diagram of the measurement setup is shown in Fig. 1. The measurement is controlled from a single standard PC running Matlab (The MathWorks Inc., Natick, MA) under Linux. The ultrasound acquisition is carried out using the experimental ultrasound scanner RASMUS [18], which is controlled from the PC through an ethernet connection. It is a realtime ultrasound system providing full control of the transducer both in transmit and receive. It is capable of storing 16 GBytes of raw ultrasound data with a sampling frequency of $40 \mathrm{MHz}$ and a precision of 12 bits for offline processing, which is essential in an experiment like this. For the acquisition, a single 64 element phased array transducer (B-K Medical, Herlev, Denmark) is connected to the RASMUS system. It has a center frequency of $3 \mathrm{MHz}$ and a $-6 \mathrm{~dB}$ bandwidth of 60 percent. The transducer is sealed to the measuring chamber giving no barrier between the contrast agent and the transducer. The measuring chamber is airtight and consists of
TABLE I

VARIOUS SETUP PARAMETERS FOR THE EXPERIMENT. FIRST PART DESCRIBES THE EXCITATION PULSE. THE SECOND PART IS RELATED TO THE EMISSION SEQUENCE. THE FINAL PART DENOTES THE AMBIENT PRESSURE SET POINTS

\begin{tabular}{|c|c|c|}
\hline Parameter & Designation & Unit \\
\hline$f_{0}$ & 4.0 & {$[\mathrm{MHz}]$} \\
\hline$N_{c}$ & 32 & [cycles] \\
\hline$P_{a c}$ & 485 & {$[\mathrm{kPa}]$} \\
\hline Shape & $10 \%$ cosine tapered & \\
\hline$N_{\text {emis }}$ & 50 & [emissions] \\
\hline$f_{p r f}$ & 50 & {$[\mathrm{~Hz}]$} \\
\hline$p_{o v}$ & $\begin{array}{llllll}0 & 5 & 10 & 15 & 20 & 25\end{array}$ & {$[\mathrm{kPa}]$} \\
\hline
\end{tabular}

two parts separated by a rubber membrane. The bottom part has a volume of $605 \mathrm{ccm}$ and can be filled with either water or saline. The walls are coated with acoustic damping material to reduce ultrasound reflections from prior emissions. It also has inlets for the transducer, fast injection of contrast agent, and a sensor to monitor the pressure within the chamber. A magnetic stirrer IKA RCT (IKA-Werke GmbH \& Co. KG, Staufen, Germany) is used to keep the bubbles in motion. The purpose of the lid, which has a dead volume of $12.5 \mathrm{ccm}$, is to change the pressure inside the chamber without mixing the inflated air with the bubbles. The pressure is managed by a custom designed dual valve pressure controller PCD4-10PSIG (Alicat Scientific, Tucson, AZ). It has an external pressure sensor and is fully programmable in real time through a RS232 serial interface connected to a PC. The compressed air is generated by a silent oil-less compressor OF301-4M (Jun-Air International A/S, Nørresundby, Denmark) providing a feed pressure of 4 bar. This is reduced to a constant feed pressure of 2 bar using a separate precision regulator from ATD Tools (Wentzville, MO).

\section{B. Experimental procedure}

The setup parameters for the measurement are listed in Table I. The acoustic bubble response was measured at six different ambient pressures between 0 and $25 \mathrm{kPa}(1 \mathrm{kPa}$ $=7.5 \mathrm{mmHg}$ ) corresponding to the common physiological blood pressure range in the human body. The measurement was initiated 90 seconds after injection of $0.5 \mathrm{ml}$ of SonoVue (Bracco, Milano, Italy) into 0.61 of saline. 50 pulses was emitted with a pulse repetition frequency of $50 \mathrm{~Hz}$ at each ambient pressure. The ambient pressure was increased in steps of $5 \mathrm{kPa}$ every 2 second until a peak ambient pressure of 25 $\mathrm{kPa}$ and was then decreased in steps of $5 \mathrm{kPa}$. The ambient pressure was allowed 1 second to adjust in between acquisition at each pressure setting. The entire measurement lasted 21 seconds and provided two sets of scattered ultrasound data at each ambient pressure, except at $25 \mathrm{kPa}$. The excitation pulse was a steered beam with an acoustic pressure of 485 $\mathrm{kPa}$ and a focus at a depth of $30 \mathrm{~mm}$ from the transducer surface. It consisted of a 32 cycles cosine tapered pulse with a center frequency of $4 \mathrm{MHz}$. The acquired data was first filtered allowing the subharmonic, $f_{\text {sub }}=2 \mathrm{MHz}$, and the 


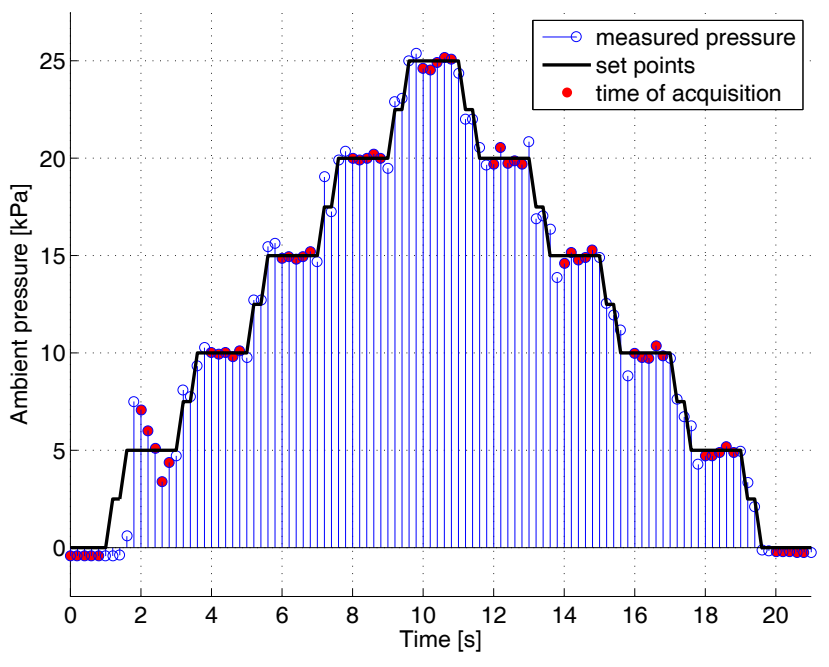

Fig. 2. Ambient pressure measured during the experiment. The stems show the pressure measured inside the chamber. The solid thick line indicates the pressure set points transmitted to the pressure controller. Finally, the dots inside the circles denote the time of ultrasound data acquisition.

fundamental, $f_{0}=4 \mathrm{MHz}$, components to pass. Next, each acquisition line was beamformed and 20 data segments of 80 samples each were extracted, using a 50 percent overlap according to Welch [19]. The periodogram was found using Bartlett's method [20] and applying a Hanning window to each segment before calculating the Fourier spectrum. Next, the energy of the subharmonic and fundamental component was calculated using a bandwidth of $0.5 \mathrm{MHz}$ centered around the respective peak amplitude. To reduce factors like UCA concentration and time dependency, the relation between the energy of the subharmonic and the fundamental is found before averaging over 10 consecutive emissions. As 50 lines are acquired, this yields 5 estimates at each ambient pressure set point.

\section{RESULTS}

A summary of the ambient pressure control during the measurement is shown in Fig. 2. It displays the instantaneous pressure, measured by the sensor inside the chamber, along with the desired pressure transmitted from the PC. The time intervals for acquiring the ultrasound data is furthermore indicated by the filled circles. The relatively large overshoot when applying an ambient over pressure for the first time is not fully understood. However, the most likely explanation is that the rubber membrane got stuck to the inlet for compressed air. To compensate, the pressure controller increases the feed pressure and, eventually, pushing the membrane downward rather powerfully. To fix this, the space between the membrane and the inlet of the feed pressure can be increased as this is only $2 \mathrm{~mm}$ in the current setup. A larger dead volume (with limitations) would probably also reduce the general ripple when changing the set point and, thereby, refine the precision and speed of the ambient pressure regulation. Disregarding the first set point at $5 \mathrm{kPa}$, the measured ambient pressure is

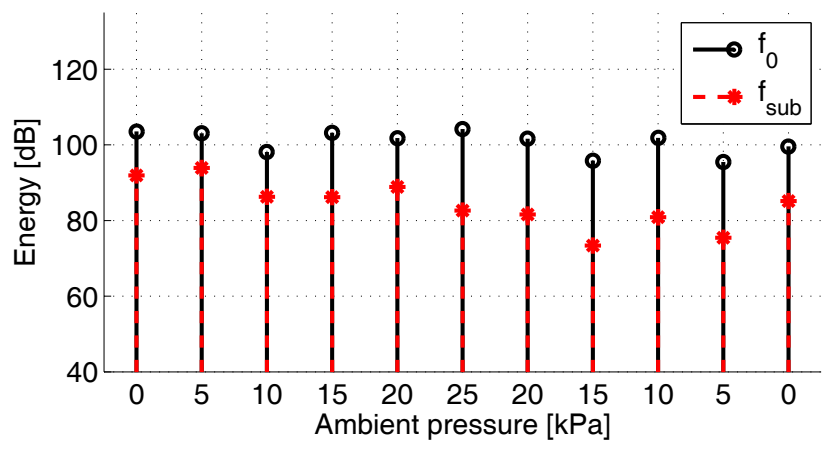

Fig. 3. Energy of the fundamental and subharmonic component estimated at the 11 different ambient pressures. Each value is the mean of five estimates which has been found based on 200 separate spectra each.

within $0.5 \mathrm{kPa}$ of the desired set points. The maximum relative deviation in respect to the desired set point is $5.8 \%$, which is observed during the second measurement at $5 \mathrm{kPa}$.

with a maximum relative deviation of $5.8 \%$, which is observed during the second measurement at $5 \mathrm{kPa}$.

Fig. 3 shows the calculated energy of the fundamental and subharmonic component as a function of ambient pressure and in order of time for the respective measurements. The energy of the fundamental component is more or less stable until about $p_{o v}=25 \mathrm{kPa}$, where it seems to start decreasing. The subharmonic component seems to drop from the beginning of the experiment to the end. According to Shi and colleagues [6], [14], this was expected for the first six measurement points. But the fact that the energy continues to drop for at least the next two measurement points $\left(p_{o v}=\left[\begin{array}{ll}20 & 15\end{array}\right] \mathrm{kPa}\right)$ could indicate that the bubbles are being dissolved. Looking at the results for pressure setting one and six constituting the first measurement series, the energy of the fundamental component changes $0.6 \mathrm{~dB}$. In the same interval, the energy of the subharmonic component is reduced by $9.2 \mathrm{~dB}$. Both these observations correspond well to the results presented in [6] and [14]. However, the fluctuating nature and the overall decrease in energy seen in Fig. 3 necessitates a more robust measure. Therefore, the relation between the energy of the subharmonic and the fundamental component is used in this experiment. The result is shown in top of Fig. 4, which also includes the standard deviation of the five estimates at each ambient pressure setting. According to Welch's method, the standard deviation scales with the number of segments used in the periodogram [19]. As 20 segments in each of 10 emissions are used for the estimate, the minimum standard deviation expected is $\sigma^{2} \approx \frac{1}{200} P_{x}^{2}(f)$. Looking at the standard deviation in Fig. 4, it is rather high compared to this. Part of the reason can be because of the low pulse repetition frequency. However, to understand the deviation fully and to improve the accuracy, a more thorough investigation regarding the choice on number of segments and emissions, as well as the $f_{p r f}$, should be carried out. Despite the high standard deviation, a clear trend can still be observed from the two measurement series in the plot in top of Fig. 4. As the ambient pressure 

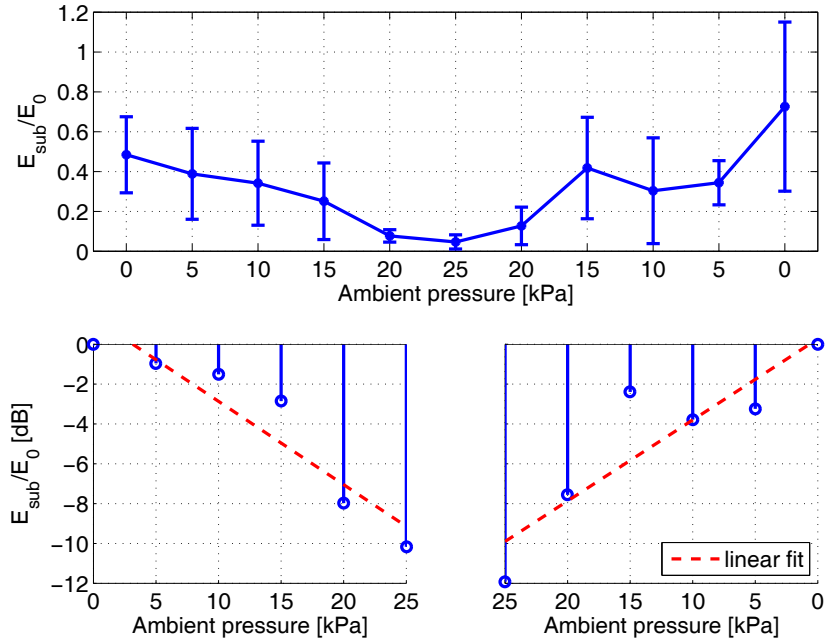

Fig. 4. The plot on top shows the relation between the energy of the subharmonic and the fundamental component estimated at each of the 11 ambient pressures. The error bars show the standard deviation which has be calculated based on five estimates. Below, the relation has been normalized and the logarithm applied for each of the two measurement series.

is increased, the relationship seems to drop. To investigate this further, each measurement series has been normalized according to its peak value at $0 \mathrm{~Pa}$ before applying the logarithm. The results are shown in the two bottom plots in Fig. 4. The dashed lines indicate a first order polynomial fit, which minimizes the error in a least-squares sense. For the first measurement series displayed to the bottom left in Fig. 4, the linear fit indicates an ambient pressure sensitivity of 0.42 $\mathrm{dB} / \mathrm{kPa}$ with a linear correlation coefficient of 0.94 . In the second measurement series, the pressure sensitivity is 0.41 $\mathrm{dB} / \mathrm{kPa}$ having a correlation coefficient of 0.89 .

\section{CONCLUSION}

An experimental measurement setup for investigating the ambient pressure sensitivity of an UCA has been designed. It consits of a single phased array transducer and equipment for automatic ambient pressure regulation and acquisition of raw ultrasound data. The setup has been used to measure the acoustic response of SonoVue when subjected to six different ambient hydrostatic pressures. The pressure management system proved capable of regulating the pressure inside the chamber within 1 second with a maximum relative deviation of $5.8 \%$. During the experiment, 21 seconds of data was acquired. As the amplitude as well as the energy of the subharmonic component was found to be useless as a measure by itself, the relationship of the energy between the subharmonic and the fundamental component was used. This yielded an ambient pressure sensitivity of 0.42 and 0.41 $\mathrm{dB} / \mathrm{kPa}$ for the two measurement series carried out. The linear correlation coefficient was 0.94 and 0.89 , respectively.

\section{ACKNOWLEDGMENT}

This work was supported by grant 26-04-0024 from the Danish Science Foundation, the Technical University of Den- mark, and by B-K Medical Aps.

\section{REFERENCES}

[1] A. C. Burton, Physiology and Biophysics of the Circulation, 2nd ed. Chicago: Year Book Medical Publishers, 1972.

[2] A. L. Strauss, F. J. Roth, and H. Rieger, "Noninvasive assessment of pressure gradients across iliac artery stenoses: duplex and catheter correlative study," J. Ultrasound Med., vol. 12, pp. 17-22, 1993.

[3] A. K. Reddy, G. E. Taffet, and S. Madala, "Noninvasive blood pressure measurement in mice using pulsed doppler ultrasound," Ultrasound Med. Biol., vol. 29, pp. 379-385, 2003.

[4] W. M. Fairbank and M. O. Scully, "A new noninvasive technique for cardiac pressure measurements: resonant scattering of ultrasound from bubbles," IEEE Trans. Biomed. Eng., vol. 24, pp. 107-110, 1977.

[5] B. Hok, "A new approach to noninvasive manometry: Interaction between ultrasound and bubbles," Med. Biol. Eng. Comp., vol. 19, pp. 35-39, 1981.

[6] W. T. Shi, F. Forsberg, J. S. Raichlen, and L. Needleman, "Pressure dependence of subharmonic signals from contrast microbubbles," Ultrasound Med. Biol., vol. 25, pp. 275-283, 1999.

[7] E. G. Tickner, "Precision microbubbles for right side intracardiac pressure and flow measurements," Meltzer RS, Roelandt JTCR, eds. Contrast echocardiography, vol. 15, pp. 313-324, 1982.

[8] K. Ishihara, A. Kitabatake, J. Tanouchi, K. Fujii, M. Uematsu, Y. Yoshida, T. Kamada, T. Tamura, K. Chihara, and K. Shirae, "New approach to noninvasive manometry based on pressure dependent resonant shift of elastic microcapsules in ultrasonic frequency characteristics," Jpn. J. Appl. Phys., vol. 27, pp. 125-127, 1988.

[9] R. Schlief and H. Poland, "Ultrasonic manometry process in a fluid by means of microbubbles, US patent number 5,195,520," March 1993.

[10] V. L. Newhouse and P. M. Shankar, "Bubble size measurements using the nonlinear mixing of two frequencies," J. Acoust. Soc. Am., vol. 75, no. 5, pp. 1473-1477, 1984.

[11] P. M. Shankar, J. Y. Chapelon, and V. L. Newhouse, "Fluid pressure measurement using bubbles insonified by two frequencies," Ultrasonics, vol. 24, pp. 333-336, November 1986.

[12] A. Bouakaz, P. J. Frinking, N. de Jong, and N. Bom, "Noninvasive measurement of the hydrostatic pressure in a fluid-filled cavity based on the dissappearance time of micrometer-sized free gas bubbles," Ultrasound Med. Biol., vol. 25, no. 9, pp. 1407-1415, 1999.

[13] A. Bouakaz, P. J. Frinking, and N. de Jong, "Noninvasive pressure measurement using microbubble contrast agent and wavelet transforms," Proc. IEEE Ultrason. Symp., pp. 1907-1910, 2000.

[14] L. M. Leodore, F. Forsberg, and W. T. Shi, "In vitro pressure estimation obtained from subharmonic contrast microbubble signals," Proc. IEEE Ultrason. Symp., 2007.

[15] F. Forsberg, J.-B. Liu, W. T. Shi, J. Furuse, M. Shimizu, and B. B. Goldberg, "In vivo pressure estimation using subharmonic contrast microbubble signals: Proof of concept," IEEE Trans. Ultrason., Ferroelec., Freq. Contr., vol. 52, pp. 581-583, 2005.

[16] D. Adam, M. Sapunar, and E. Burla, "On the relationship between encapsulated ultrasound contrast agent and pressure," Ultrasound Med. Biol., vol. 31, pp. 673-686, 2005.

[17] K. S. Andersen and J. A. Jensen, "Simulation of microbubble response to ambient pressure changes," Med. Imag. V Symp., vol. 6920, p. 692016, 2008.

[18] J. A. Jensen, O. Holm, L. J. Jensen, H. Bendsen, H. M. Pedersen, K. Salomonsen, J. Hansen, and S. Nikolov, "Experimental ultrasound system for real-time synthetic imaging," in Proc. IEEE Ultrason. Symp., vol. 2, 1999, pp. 1595-1599.

[19] P. D. Welch, "The use of fast Fourier transform for the estimation of power spectra: A method based on time averaging over short, modified periodograms," IEEE Trans. Au. Electroacous., vol. AU-15, pp. 70-73, 1967.

[20] M. S. Bartlett, "Smoothing periodograms from time series with continuous spectra," Nature (London), vol. 161, pp. 686-687, May 1948. 